\title{
Origens e ramificações do plexo braquial do veado catingueiro Mazama gouazoubira (Artiodactyla: Cervidae)
}

\author{
Lucélia Gonçalves Vieira \\ Priscilla Rosa Queiroz Ribeiro * \\ Mariana Oliveira Lima \\ Rogério Rodrigues de Souza \\ Sady Alexis Chavauty Valdes \\ André Luiz Quagliatto Santos
}

Laboratório de Ensino e Pesquisa em Animais Silvestres

Universidade Federal de Uberlândia, CEP 38408-078, Uberlândia - MG, Brasil

* Autor para correspondência

priscillarqr@yahoo.com.br

Submetido em 28/08/2012

Aceito para publicação em 28/11/2012

\section{Resumo}

O plexo braquial é um conjunto de nervos com origem na região medular cervicotorácica que inerva o membro torácico e adjacências. Seu estudo em diferentes espécies é importante não apenas como uma fonte de conhecimento morfológico, mas, também, por facilitar o diagnóstico de disfunções neuromusculares decorrentes de diversas patologias. O objetivo foi descrever as origens e ramificações do plexo braquial do Mazama gouazoubira. Foram utilizados três espécimes, pertencentes ao acervo científico do Laboratório de Ensino e Pesquisa em Animais Silvestres da Universidade Federal de Uberlândia (UFU); eles foram fixados em formaldeído a 3,7\% e dissecados. No M. gouazoubira, o plexo braquial resultou de conexões entre os ramos dos três últimos nervos espinhais cervicais, $\mathrm{C} 6, \mathrm{C} 7$ e C8, e do primeiro torácico T1, e teve como derivações os nervos supraescapular, subescapulares cranial e caudal, axilar, musculocutâneo, mediano, ulnar, radial, peitorais, toracodorsal, torácico longo e torácico lateral. Os músculos inervados pelos nervos do plexo braquial foram o supraespinhal, infraespinhal, subescapular, redondo maior, redondo menor, deltoide, cleidobraquial, coracobraquial, bíceps braquial, braquial, tríceps braquial, ancôneo, flexor superficial dos dedos, flexor profundo dos dedos, flexor radial do carpo, flexor ulnar do carpo, extensor radial do carpo, ulnar lateral, extensor oblíquo do carpo, extensores dos dedos, peitoral superficial, peitoral profundo, serrátil ventral e oblíquo externo do abdome.

Palavras-chave: Anatomia; Cervídeos; Músculos; Nervos

\section{Abstract}

Origins and branchings of the brachial plexus of the gray brocket deer Mazama gouazoubira (Artiodactyla: Cervidae). The brachial plexus is a set of nerves originated in the cervicothoracic medular region which innervates the thoracic limb and its surroundings. Its study in different species is important not only as a source of morphological knowledge, but also because it facilitates the diagnosis of neuromuscular disorders 
resulting from various pathologies. This study aimed to describe the origins and branchings of the brachial plexus of Mazama gouazoubira. Three specimens were used, belonging to the scientific collection of the Laboratory for Teaching and Research on Wild Animals of Universidade Federal de Uberlandia (UFU); they were fixed in $3.7 \%$ formaldehyde and dissected. In M. gouazoubira, the brachial plexus resulted from connections between the branches of the three last cervical spinal nerves, C6, C7, C8, and the first thoracic one, T1, and it had as derivations the nerves suprascapular, cranial and caudal subscapular, axillary, musculocutaneous, median, ulnar, radial, pectoral, thoracodorsal, long thoracic and lateral thoracic. The muscles innervated by the brachial plexus nerves were the supraspinatus, infraspinatus, subscapularis, teres major, teres minor, deltoid, cleidobrachial, coracobrachialis, biceps brachialis, brachial, triceps brachialis, anconeus, flexor digitorum superficialis, flexor digitorum profundus, flexor carpi radialis, flexor carpi ulnaris, extensor carpi radialis, lateral ulnar, extensor carpi obliquus, extensor digitorum, superficial pectoral, deep pectoral, ventral serratus, and external oblique abdominal.

Key words: Anatomy; Cervids; Muscles; Nerves

\section{Introdução}

Os cervídeos constituem um grupo de animais pertencentes à ordem Artiodactyla e à família Cervidae. Seus diferentes gêneros, endêmicos da América do Sul, são Blastocerus, Hippocamelus, Mazama, Odocoileus, Ozotoceros e Pudu. O Mazama gouazoubira, conhecido popularmente como veado catingueiro, é uma espécie de pequeno porte que pode ser encontrada na América do Sul, desde o sul do Uruguai até o norte de Mato Grosso, no Brasil (FERREIRA et al., 2011). É um cervo de coloração marrom acinzentada e com cauda branca na face ventral, possui chifres pequenos e simples e glândulas de odor característico caudais aos olhos e nos garrões (LIMA et al., 2010).

O plexo braquial é um conjunto de nervos com origens nos segmentos cervicotorácicos da medula, que se distribui pelos membros torácicos e porção interna do tórax (SCAVONE et al., 2008). Segundo Miller et al. (1964), Dyce et al. (1996) e Ghoshal (1996), o plexo braquial, nas espécies domésticas, é composto por fibras neurais sensitivas e motoras que inervam o membro torácico, geralmente formado pelo fornecimento dos ramos ventrais dos três últimos nervos cervicais e dois primeiros nervos torácicos. O quinto nervo cervical em alguns casos participa e a contribuição do segundo nervo torácico é reduzida ou ausente.

Estes ramos combinam-se formando os troncos cranial, constituído por $\mathrm{C} 4, \mathrm{C} 5$ e $\mathrm{C} 6$, ou um tronco comum, entre $\mathrm{C} 4$ e C5, com posterior ligação do C6; o tronco médio formado somente por $\mathrm{C} 7$; e o tronco caudal constituído por C8 e T1 e menos frequentemente por T2 (MILLER, 1934; KOIZUMI; SAKAI, 1995). O plexo passa pela axila entre as partes do músculo escaleno e se divide em ramos periféricos que divergem em direção aos seus respectivos territórios.

Ao consultar a literatura especializada poucos trabalhos sobre a morfologia funcional dos cervídeos foram encontrados como os aspectos morfológicos do fígado do cervo do pantanal (BORGES et al., 2002), sistema excretor do fígado de veados (MIGLINO et al., 1995), componentes do funículo espermático do veado mateiro (BORELLI; GONÇALVES, 2011), morfologia das membranas fetais e da placenta (FERREIRA et al., 2011) e morfologia do testículo (COSTA, 2009) de $M$. gouazoubira. No que diz respeito ao sistema neural, esse número é ainda menor, destacando-se os trabalhos de topografia vértebro-medular (LIMA et al., 2010) e de organização macroestrutural do plexo braquial relacionado ao sexo (MELO et al., 2007) em M. gouazoubira.

Existem vários trabalhos para outros mamíferos, que abordam a origem, ramificação e distribuição do plexo braquial. Isto tem sido demonstrado nas contribuições em Hydrochaeris hydrochaeris (FIORETTO et al., 2003), Kerodon rupestris (SANTANA et al., 2003), Bradypus variegatus (AMORIM JÚNIOR et al., 2003), Chinchilla lanigera (GAMBA et al., 2007), Tayassu tajacu (MOURA et al., 2007), Agouti paca (SCAVONE et al., 2008), Arctocephalus australis (SOUZA et al., 2010), porém no M. gouazoubira há escassez de informações sobre essa abordagem. 
O estudo do plexo braquial em diferentes espécies é importante, não apenas como um conhecimento morfológico, mas também por facilitar o diagnóstico de disfunções neuromusculares, decorrentes de diversas patologias (FREEMAN et al., 2009). Além disso, o conhecimento da disposição anatômica do plexo braquial, bem como dos músculos por ele inervados, permite a aplicação adequada da técnica anestésica de bloqueio do plexo braquial (FUTEMA et al., 1999). Esta técnica de anestesia regional vem sendo descrita na literatura desde o início do século XX (STRODE, 1929) e vem até hoje sendo adaptada a diversas espécies domésticas (IWAMOTO et al., 2012, RIOJA et al., 2012), porém há poucos relatos em espécies selvagens (BRENNER et al., 2010).

Assim, o objetivo dessa pesquisa foi descrever as origens e ramificações dos nervos que compõem o plexo braquial, bem como os músculos por eles inervados, no M. gouazoubira.

\section{Material e Métodos}

Para o desenvolvimento deste trabalho foram utilizados três espécimes adultos, dois machos e uma fêmea, de M. gouazoubira Fisher (1814), pertencentes ao acervo científico do Laboratório de Ensino e Pesquisa em Animais Silvestres (LAPAS), da Universidade Federal de Uberlândia (UFU).

Os animais estudados foram fixados em solução aquosa de formaldeído a 3,7\% e, posteriormente, conservados imersos em cubas opacas contendo solução de igual concentração. A preparação dos espécimes para análise seguiu as técnicas anatômicas propostas por Rodrigues (2005).

Após a remoção da pele e de parte do tecido adiposo, rebateram-se as fáscias da região braquial e posteriormente foi realizada a dissecação bilateral das origens dos nervos e suas ramificações. A nomenclatura adotada para descrição dos resultados se baseou na Nomina Anatomica Veterinaria (ICVGAN, 2005).

Para a fotodocumentação dos resultados utilizou-se uma câmera fotográfica digital (Sony DSC H20 10.1).

\section{Resultados}

No M. gouazoubira, o plexo braquial resultou de conexões entre os ramos dos três últimos nervos espinhais cervicais (C6, C7 e C8) e primeiro torácico (T1). Teve como derivações os nervos supraescapular, subescapulares cranial e caudal, axilar, musculocutâneo, mediano, ulnar, radial, peitorais, toracodorsal, torácico longo e torácico lateral (Figura 1).

O nervo supraescapular originou-se dos nervos C6 e C7. Em seu trajeto passou entre os músculos supraespinhal e subescapular, emitindo ramos a ambos, de onde seguiu em direção ao acrômio da escápula e correu sob o mesmo, para inervar o músculo infraespinhal (Figura 1).

Com origem nos nervos espinhais $\mathrm{C} 6$ e $\mathrm{C} 7$, o nervo subescapular dividiu-se em nervos subescapulares cranial e caudal. O nervo subescapular cranial partiu do plexo braquial em companhia do nervo supraescapular e penetrou na parte cranial do músculo subescapular. $\mathrm{O}$ nervo subescapular caudal acompanhou o nervo axilar e penetrou na parte caudal do músculo subescapular e no redondo maior (Figura 1).

O nervo axilar foi constituído por fibras dos nervos C6 e C7, situando-se lateralmente, entre os espaços dos músculos subescapular, redondo maior e cabeça longa do tríceps braquial, cruzando caudalmente à articulação do ombro. Antes de penetrar entre os músculos supracitados esse nervo emitiu ramos para a extremidade distal dos músculos subescapular e redondo menor, outro ramo seguiu entre a parte acromial do deltóide, para o qual emitiu ramo, e o tubérculo maior do úmero (Figura 1).

Formado por fibras dos nervos $\mathrm{C} 6, \mathrm{C} 7 \mathrm{e} \mathrm{C} 8$, o nervo musculocutâneo cruzou a face medial da artéria axilar e uniu-se ao nervo mediano, com o qual constituiu a alça axilar, região a qual emitiu o ramo muscular proximal do nervo musculocutâneo que foi responsável pela inervação dos músculos coracobraquial e bíceps braquial. Este nervo continuou distalmente no braço unido ao nervo mediano. Ao nível do terço distal do braço se separou do nervo mediano e emitiu o ramo muscular distal do nervo musculocutâneo, responsável pela inervação do músculo braquial e continuou como ramo cutâneo medial do antebraço, este responsável pelo aporte nervoso à pele da face medial deste segmento (Figura 1). 
FIGURA 1: Origem e ramificação do plexo braquial de Mazama gouazoubira adulto em vista lateral. C6, C7, C8, segmentos cervicais 6, 7 e 8; $\mathbf{T}_{1}$, segmento torácico $1 ; \mathbf{m b}$, músculo bíceps braquial; $\mathbf{m g d}$, músculo grande dorsal; mrm, músculo redondo maior; mse, músculo subescapular; msp, músculo supraescapular; mtl, cabeça longa do músculo tríceps braquial; nax, nervo axilar; nme, nervo mediano; nra, nervo radial; nse, nervo subescapular; nseca, nervo subescapular caudal; nsecr, nervo subescapular cranial; nsp, nervo supraescapular; ntd, nervo toracodorsal; ntlo, nervo torácico longo; nul, nervo ulnar; rntla, ramo do nervo torácico lateral; rdmc, ramo muscular distal do nervo musculocutâneo; rpmc, ramo muscular proximal do nervo musculocutâneo.

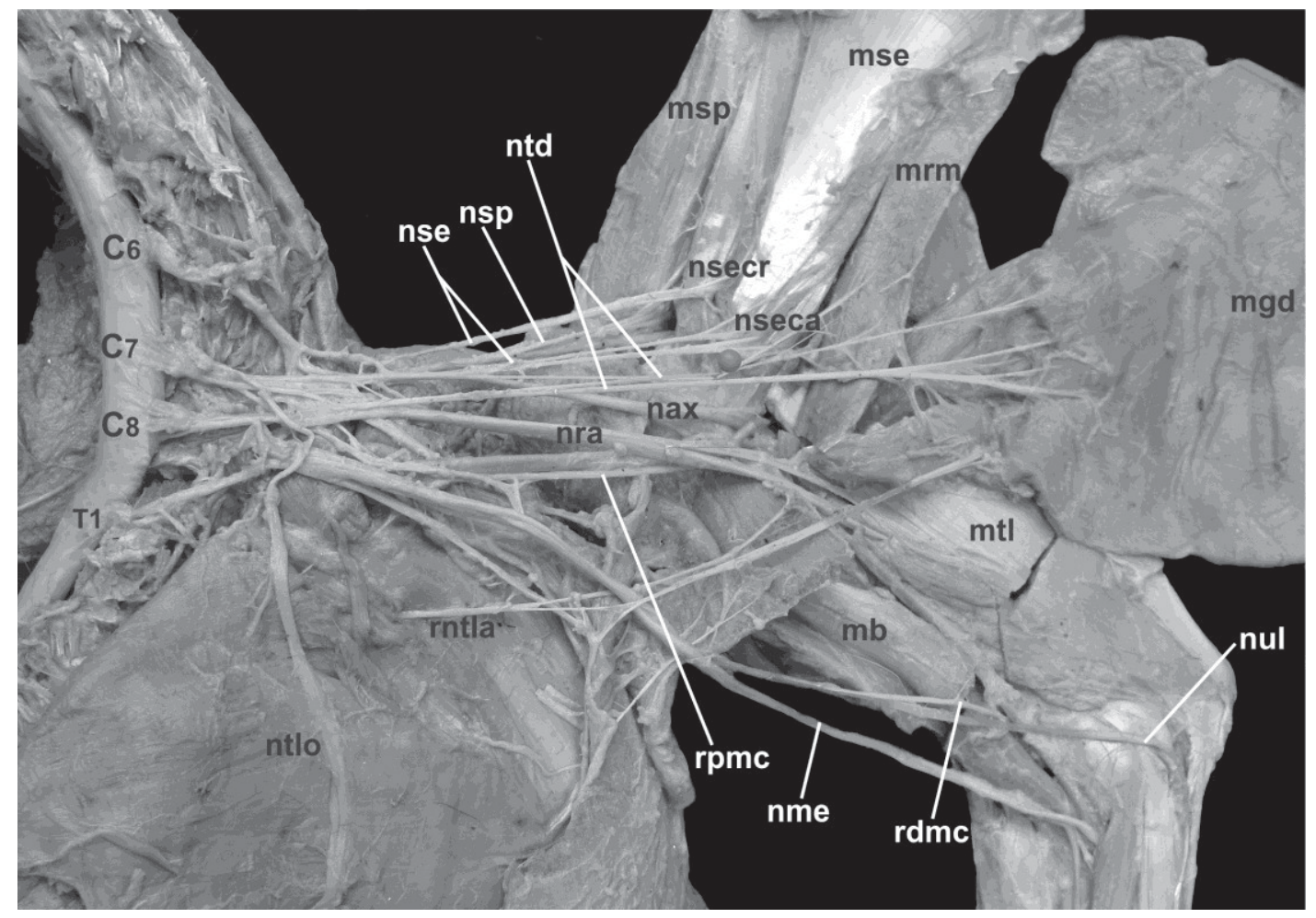

O nervo mediano originou-se dos nervos $\mathrm{C} 7, \mathrm{C} 8$ e T1, cruzou a face medial da artéria axilar e correu distalmente na face medial do braço caudalmente aos músculos coracobraquial e bíceps braquial. Ao nível da face medial da articulação do cotovelo, aproximou-se da artéria braquial e correu profundamente às inserções dos músculos braquiocefálico e parte descendente do peitoral superficial e emitiu forte ramo que, após curto trajeto, ramificou-se na face profunda dos músculos pronador redondo, flexor radial do carpo, flexor superficial dos dedos e nas cabeças umerais do flexor profundo dos dedos. Na extremidade proximal do antebraço, passou profundamente ao músculo pronador redondo em companhia da artéria braquial, onde forneceu ramos à cabeça radial do músculo flexor profundo dos dedos e o ramo interósseo do antebraço. A partir daí prosseguiu distalmente o seu curso na face medial do antebraço, profundamente ao músculo flexor radial do carpo, próximo à artéria mediana. $\mathrm{Na}$ extremidade distal do antebraço, a artéria e o nervo tornaram-se superficiais e passaram entre os tendões dos músculos flexor radial do carpo e superficial dos dedos e continuaram-se na face palmar, onde o nervo enviou vários ramos (Figura 1).

Com origem nos nervos C8 e T1, na região axilar, o nervo ulnar seguiu em companhia da artéria axilar. Ao nível do terço médio do braço inclinou-se caudalmente e cruzou a artéria braquial, onde emitiu, na face medial da cabeça longa do tríceps braquial, o nervo cutâneo cranial do antebraço, responsável pela inervação da fáscia e pele dessa região. À altura da articulação do cotovelo passou profundamente entre as origens umeral e ulnar do músculo flexor ulnar do carpo, onde enviou ramos para este, para o flexor superficial dos dedos e para as cabeças umeral e ulnar do músculo flexor profundo dos dedos. Continuou seu trajeto distal passando pela margem caudal do antebraço entre os músculos extensor ulnar do carpo 
e flexor ulnar do carpo e se dividiu em ramos palmar e dorsal, ao nível do terço distal do antebraço (Figura 1).

Formado a partir dos nervos $\mathrm{C} 7 \mathrm{e} \mathrm{C} 8$, o nervo radial relacionou-se lateralmente com as artérias subescapular, toracodorsal e braquial. Ao atingir o espaço compreendido entre o músculo redondo maior e as cabeças medial e longa do tríceps braquial, forneceu fibras a estas cabeças e ao músculo tensor da fáscia do antebraço. A partir de então continuou correndo distal e lateralmente, passou entre as cabeças medial e acessória do tríceps braquial, para alcançar o sulco do músculo braquial. Neste percurso situou-se entre o músculo braquial e a cabeça lateral do tríceps braquial, onde emitiu ramos para esta cabeça e para o músculo ancôneo. Em um ponto variável, proximal à articulação do cotovelo, dividiu-se em ramos superficial e profundo. $\mathrm{O}$ ramo profundo passou distalmente entre os músculos braquial e extensor radial do carpo, onde enviou ramos para estes dois músculos e continuou seu trajeto passando entre o músculo extensor radial do carpo e o colo do rádio. O ramo superficial seguiu distalmente, cruzou a face lateral do terço distal do músculo braquial e atingiu a face cranial do antebraço, onde correu na fáscia antebraquial e emitiu a partir daí vários ramos para a pele e fáscias durante seu trajeto no antebraço e mão (Figura 1).

Os nervos peitorais apresentaram tronco comum com o nervo torácico lateral e se originaram de fibras dos nervos C8 e T1. As fibras de T1 passaram por uma abertura no músculo serrátil ventral e dividiram-se em ramos craniais, que inervaram as partes craniais dos músculos peitoral superficial e peitoral profundo e em ramos caudais, que inervaram a parte caudal dos mesmos músculos. Com a mesma origem dos nevos peitorais o nervo torácico lateral passou caudalmente e se distribuiu pelo músculo grande dorsal, parte caudal do peitoral profundo e oblíquo externo do abdome (Figura 2).

$\mathrm{O}$ nervo toracodorsal constituiu-se por fibras dos nervos $\mathrm{C} 7$ e $\mathrm{C} 8$ e bifurcou-se para emitir ramos para os músculos grande dorsal e redondo maior (Figura 2).

O nervo torácico longo teve sua origem nas fibras dos nervos $\mathrm{C} 7$ e $\mathrm{C} 8$ de onde correu junto ao músculo serrátil ventral, inervando-o (Figura 2).

FIGURA 2: Nervos torácicos longo e lateral e peitoral profundo de Mazama gouazoubira adulto em vista lateral. moe, músculo obliquo externo do abdome; mpp, músculo peitoral profundo; msv, músculo serrátil ventral; npca, nervo peitoral caudal; npcr, nervo peitoral cranial; ntla, nervo torácico lateral; ntlo, nervo torácico longo.

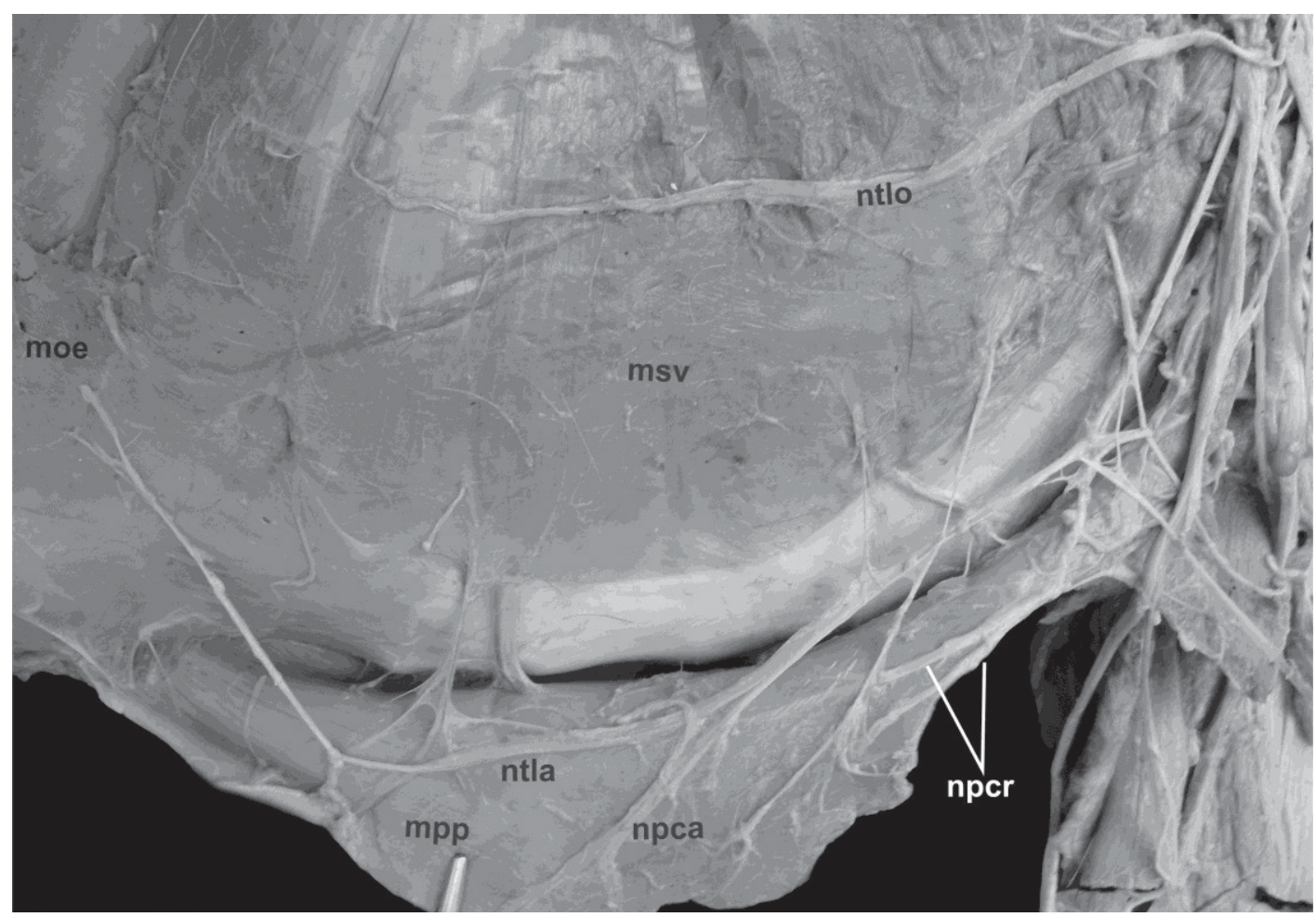




\section{Discussão}

O plexo braquial do $M$. gouazoubira originouse de uniões dos nervos espinhais C6, C7, C8 e T1, assim como nos ovinos e caprinos (GOSHAL, 1986). Nos bovinos, sua origem abrange também o nervo T2 (GOSHAL, 1986). Nas espécies não ruminantes, Ornithorhynchus anatinus (KOIZUMI; SAKAI, 1997), Cebus apella (RIBEIRO et al., 2005), Tayassu tajacu (MOURA et al., 2007), Hydrochaeris hydrochaeris (FIORETTO et al., 2003), além dos nervos supracitados abrange também os nervos $\mathrm{C} 4$ e C5, exceto na última espécie que não envolve T2. Em Kerodon rupestris (SANTANA et al., 2003) e Agouti paca (SCAVONE et al., 2008), o nervo C4 não contribui para a constituição do plexo braquial e em Rattus norvegicus (UZUN et al., 2001), Pan troglodytes, Gorilla gorilla, Hylobates agilis, Hylobates syndactylus (KOIZUMI; SAKAI, 1995), além de C4, o nervo T2 também não participa da formação do plexo.

Em concordância com as informações de MOURA et al. (2007), acredita-se que as variações nos níveis de origem do plexo braquial constatadas acima podem estar relacionadas com fatores embriológicos, representados pela posição na qual os brotos dos membros se desenvolvem em relação ao neuroeixo. Assim, quanto mais cefálica for a relação, mais craniais serão os ramos que participam da formação do plexo.

Com origem em C6 e C7 no M. gouazoubira, o nervo supraescapular originou-se de C5, C6 e C7 na A. paca (SCAVONE et al., 2008), de C4, C5, C6 e C7 no T. tajacu (MOURA et al., 2007) e na Hydrochaeris hydrochaeris (FIORETTO et al., 2003), de C6, C7 e C8 em fetos de bovinos azebuados (SANTOS et al., 2011), de C6 na Chinchilla lanigera (GAMBA et al., 2007) e em alguns casos nos caprinos (GETTY, 1981) e de C6 e C7 no Arctocephalus australis (SOUZA et al., 2010) e nos ruminantes (GETTY, 1981). Assim como na $A$. paca (SCAVONE et al., 2008), fetos de bovinos azebuados (SANTOS et al., 2011) e nos ruminantes (GETTY, 1981), este nervo emitiu ramos para os músculos supraespinhal e infraespinhal e diferente do que ocorreu nestas espécies o músculo subescapular no $M$. goazoubira e $H$. hydrochaeris (FIORETTO et al., 2003) também recebeu ramos deste nervo. No A. australis (SOUZA et al., 2010) se restringiu à inervação dos músculos subescapular e supraespinhal. Já na C. lanigera (GAMBA et al., 2007), além dos músculos supraespinhal e infraespinhal, o músculo deltóide também foi inervado.

No M. gouazoubira, os nervos subescapulares cranial e caudal tiveram origem em $\mathrm{C} 6$ e C7, bem como na $C$. lanigera (GAMBA et al., 2007) e ruminantes (GETTY, 1981). Origens diferentes foram observadas na $A$. paca (SCAVONE et al., 2008) em que surgiu apenas de C6, e no A. australis (SOUZA et al., 2010) apenas de C7. Este último também pode ocorrer em alguns casos nos pequenos ruminantes (GETTY, 1981). O T. tajacu (MOURA et al., 2007) envolve além dos nervos observados no M. gouazoubira, o nervo C5. Este nervo foi responsável pelo aporte nervoso aos músculos subescapular e redondo maior no $M$. gouazoubira, $C$. lanigera (GAMBA et al., 2007) e A. australis (SOUZA et al., 2010), o que não foi verificado por Scavone et al. (2008) em seu trabalho com A. paca e por Getty (1981) nos ruminantes nos quais realizou a inervação apenas do músculo subescapular.

Assim como no $M$. gouazoubira, no $T$. tajacu (MOURA et al., 2007), na A. paca (SCAVONE et al., 2008), na $C$. lanigera (GAMBA et al., 2007) e nos caprinos e ovinos (GETTY, 1981), o nervo axilar se originou em C6 e C7. Já na H. hydrochaeris (FIORETTO et al., 2003), sua origem aconteceu em C5, C6, C7 e C8 e no A. australis (SOUZA et al., 2010) e bovinos (GETTY, 1981) se restringiu aos nervos C7 e C8. A área de inervação muscular desse nervo é bastante variável entre as espécies. No M. gouazoubira foi responsável pela inervação dos músculos subescapular, redondo menor e deltóide. $\mathrm{Na} A$. paca (SCAVONE et al., 2008) e ruminantes (GETTY, 1981), além destes foi responsável também pela inervação do músculo redondo maior, na H. hydrochaeris (FIORETTO et al., 2003) apenas a cabeça medial do tríceps braquial, na C. lanigera (GAMBA et al., 2007) o redondo maior, deltóide, subescapular, braquiocefálico e cleidobraquial e no A. australis (SOUZA et al., 2010) o redondo maior, deltóide e subescapular.

No M. gouazoubira o nervo musculocutâneo originou-se em C6, C7 e C8, assim como na $A$. paca 
(SCAVONE et al., 2008) e bovinos (GETTY, 1981). Já no T. tajacu (MOURA et al., 2007) a origem deu-se em C7, C8 e T1, na C. lanigera (GAMBA et al., 2007) e ovinos e caprinos (GETTY, 1981) em C6 e C7 e no A. australis (SOUZA et al., 2010) em C8 e T1. Tanto no $M$. gouazoubira como na $A$. paca (SCAVONE et al., 2008) e ruminantes (GETTY, 1981), os músculos inervados pelo ramo muscular proximal desse nervo foram o coracobraquial e bíceps braquial, já na $C$. lanigera (GAMBA et al., 2007) e A. australis (SOUZA et al., 2010) apenas o bíceps braquial. O ramo muscular distal forneceu aporte nervoso ao músculo braquial no M. gouazoubira, na A. paca (SCAVONE et al., 2008) e ruminantes (GETTY, 1981) e além deste ao músculo bíceps braquial na $C$. lanigera (GAMBA et al., 2007) e A. australis (SOUZA et al., 2010).

O nervo mediano originou-se em C7, C8 e T1 no $M$. gouazoubira, bem como na A. paca (SCAVONE et al., 2008) e C. lanigera (GAMBA et al., 2007). No T. tajacu (MOURA et al., 2007) além dos nervos supracitados abrangeu também T2, na H. hydrochaeris (FIORETTO et al., 2003) C6, C7, C8 e T1, no A. australis (SOUZA et al., 2010) e ovinos e caprinos (GETTY, 1981) C8 e T1 e nos bovinos (GETTY, 1981) C8, T1 e T2. No $M$. gouazoubira, na C. lanigera (GAMBA et al., 2007) e no A. australis (SOUZA et al., 2010) o nervo mediano foi responsável pela inervação dos músculos pronador redondo, flexores superficial e profundo dos dedos e ainda o flexor radial do carpo. Nos ruminantes (GETTY, 1981), além destes também o músculo flexor ulnar do carpo.

O nervo ulnar originou-se em $\mathrm{C} 8$ e $\mathrm{T} 1$ no $M$. gouazoubira, assim como nos ovinos e caprinos (GETTY, 1981). Na A. paca (SCAVONE et al., 2008), A. australis (SOUZA et al., 2010) e bovinos (GETTY, 1981) teve origem em C8, T1 e T2, no T. tajacu (MOURA et al., 2007) em C6, C7, C8 e T1 e na $H$. hydrochaeris (FIORETTO et al., 2003) e C. lanigera (GAMBA et al., 2007) em C7, C8 e T1. Os músculos flexor ulnar do carpo, flexor superficial dos dedos e flexor profundo dos dedos foram inervados por este nervo no M. gouazoubira e ruminantes (GETTY, 1981). Nesses últimos, inconstantemente emitiu ramos também para o extensor ulnar do carpo. $\mathrm{Na} \mathrm{H}$. hydrochaeris
(FIORETTO et al., 2003), realizou a inervação dos músculos flexor radial do carpo, flexor ulnar do carpo e flexor superficial dos dedos, na C. lanigera (GAMBA et al., 2007) do flexor radial do carpo e flexor profundo dos dedos e no A. australis (SOUZA et al., 2010) do flexor ulnar do carpo, flexor profundo dos dedos e flexores dos dedos I e II. Já na A. paca (SCAVONE et al., 2008), os autores apenas afirmaram que se distribui pelos músculos do antebraço, não os especificando.

No M. gouazoubira, o nervo radial originou-se de C7 e C8, no T. tajacu (MOURA et al., 2007) de C8, T1 e T2, na $A$. paca (SCAVONE et al., 2008), $C$. lanigera (GAMBA et al., 2007), A. australis (SOUZA et al., 2010) e ruminantes (GETTY, 1981) de C7, C8 e T1 e na H. hydrochaeris (FIORETTO et al., 2003) e Felis catus domesticus (GUIMARÃES et al., 2007) de C6, C7, C8 e T1. Foi responsável pela inervação dos músculos tríceps braquial, tensor da fáscia do antebraço, braquial, ancôneo, extensor radial do carpo, extensores dos dedos, ulnar lateral e extensor oblíquo do carpo no M. gouazoubira e bovinos (GETTY, 1981), nesses últimos, apenas em alguns casos, observou-se ramo para o músculo braquial. Na A. paca (SCAVONE et al., 2008) e ovinos e caprinos (GETTY, 1981), forneceu ramos para os mesmos músculos acima, exceto o braquial. Na C. lanigera (GAMBA et al., 2007) e A. australis (SOUZA et al., 2010) todos os músculos supracitados foram inervados, além dos músculos extensores digitais comum e lateral, oblíquo longo e os supinadores longo e breves. Na H. hydrochaeris (FIORETTO et al., 2003) os autores relataram apenas os músculos tríceps braquial, ancôneo, extensor radial do carpo e extensores dos dedos e no Felis catus domesticus (GUIMARÃES et al., 2007) somente os músculos tensor da fáscia do antebraço, tríceps braquial e ancôneo.

Os nervos peitorais originaram-se de $\mathrm{C} 8$ e $\mathrm{T} 1$ no M. gouazoubira, C7 e C8 no T. tajacu (MOURA et al., 2007) e ruminantes (GETTY, 1981), C5, C6, C7, C8 e T1 na $A$. paca (SCAVONE et al., 2008), C6, C7, C8 e T1 no A. australis (SOUZA et al., 2010) e em alguns casos nos caprinos (GETTY, 1981) e C7, C8 e T1 na $C$. lanigera (GAMBA et al., 2007) e em menor proporção nos bovinos (GETTY, 1981). Foi responsável pela inervação dos músculos peitorais superficial e profundo 
no M. gouazoubira, A. paca (SCAVONE et al., 2008), A. australis (SOUZA et al., 2010), C. lanigera (GAMBA et al., 2007) e ruminantes (GETTY, 1981).

O nervo toracodorsal originou-se de $\mathrm{C} 7$ e $\mathrm{C} 8$ no M. gouazoubira, bovinos e em alguns casos nos ovinos (GETTY, 1981). Este autor relatou ainda origem em C8 e T1 para os bovinos, porém em menor proporção. No T. tajacu (MOURA et al., 2007) surgiu de C6, C7 e C8, na $A$. paca (SCAVONE et al., 2008) de C8, T1 e T2, na H. hydrochaeris (FIORETTO et al., 2003) de C6, C7, C8 e T1, em fetos de bovinos azebuados (SANTOS et al., 2010) de $\mathrm{C} 8$ e T1, no A. australis (SOUZA et al., 2010) de C6, na C. lanigera (GAMBA et al., 2007) e nos ovinos e caprinos (GETTY, 1981) de C8. Este nervo foi responsável pela inervação dos músculos grande dorsal e redondo maior no M. gouazoubira, fetos de bovinos azebuados (SANTOS et al., 2010) e ovinos (GETTY, 1981), nestes dois últimos também pelo peitoral profundo. Na A paca (SCAVONE et al., 2008), H. hydrochaeris (FIORETTO et al., 2003), A. australis (SOUZA et al., 2010), C. lanigera (GAMBA et al., 2007), bovinos e caprinos (GETTY, 1981) foi responsável pela inervação apenas do grande dorsal.

A origem do nervo torácico longo ocorreu em C7 e C8 no M. gouazoubira, à semelhança de $A$. australis (SOUZA et al., 2010), C. lanigera (GAMBA et al., 2007), A. paca (SCAVONE et al., 2008), T. tajacu (MOURA et al., 2007), ruminantes (GETTY, 1981) e H. hydrochaeris (FIORETTO et al., 2003). Nesta última espécie incluiu também o ramo de C6. Em todas as espécies citadas este nervo foi responsável pelo aporte nervoso do músculo serrátil ventral, exceto na $A$. paca (SCAVONE et al., 2008), onde envia ramos somente ao grande dorsal.

No M. gouazoubira, C. lanigera (GAMBA et al., 2007), A. australis (SOUZA et al., 2010), ovinos e caprinos (GETTY, 1981) o nervo torácico lateral emergiu dos nervos C8 e T1. No T. tajacu (MOURA et al., 2007), A. paca (SCAVONE et al., 2008) e bovinos (GETTY, 1981) incluiu também o T2. Na H. hydrochaeris (FIORETTO et al., 2003) não houve contribuição de T2, no entanto, C7 participou da formação deste nervo. No M. gouazoubira responsabilizou-se pela inervação dos músculos grande dorsal, peitoral profundo e oblíquo externo do abdome, diferente do que ocorreu na $C$. lanigera (GAMBA et al., 2007), A. australis (SOUZA et al., 2010), A. paca (SCAVONE et al., 2008), H. hydrochaeris (FIORETTO et al., 2003) e ruminantes (GETTY, 1981), nos quais realizou a inervação do músculo cutâneo do tronco.

No M. gouazoubira, o plexo braquial teve origem do sexto, sétimo e oitavo nervos espinhais cervicais e do primeiro nervo espinhal torácico. O plexo derivou-se em 11 nervos (supraescapular, subescapulares cranial e caudal, axilar, musculocutâneo, mediano, ulnar, radial, peitorais, toracodorsal, torácico longo e torácico lateral), responsáveis pela inervação dos músculos da cintura escapular, braço, antebraço e parte do tronco.

\section{Agradecimentos}

Nossos agradecimentos a Caio Henrique Ferreira e Sérgio Rodrigo Pereira Oliveira por sua contribuição para o desenvolvimento do trabalho.

\section{Referências}

AMORIM JÚNIOR, A. A.; AMORIM, M. J. A. A. L.; SILVA, D. R.; PIMENTEL, D. S.; ARAÚJO F. P.; ALVIM, M. M. S. Origem do plexo braquial no bicho-preguiça (Bradypus variegatus Shinz, 1825). International Journal of Morphology, Temuco, v. 1, n. 21 p. $357-362,2003$

BORELLI, V.; GONÇALVES, A. M. M. Aspectos morfológicos dos componentes do funículo espermático do veado-mateiro (Mazama americana). Journal of the Health Sciences Institute, Mirandopolis, v. 29, n. 4, p. 294-298, 2011.

BORGES, E. M.; MACHADO, M. R. F.; OLIVEIRA, F. S.; SOUZA, W. M.; DUARTE, J. M. B. Aspectos morfológicos do fígado do cervo-do-pantanal (Blastocerus dichotomus). Brazilian Journal of Veterinary Research and Animal Science, São Paulo, v. 39 , n. 2 , p. $78-80,2002$

BRENNER, D. J.; LARSEN, R. S.; DICKINSON, P. J.; WACK, R. F.; WILLIAMS, D. C.; PASCOE, P. J. Development of an avian brachial plexus nerve block technique for perioperative analgesia in mallard ducks (Anas platyrhynchos). Journal of Avian Medicine and Surgery, Boca Raton, v. 24, n. 1, p. 24-34, 2010.

COSTA, K. L. C. Avaliação morfofuncional do testículo de veado catingueiro (Mazama gouazoubira Fisher, 1814). 2009. 87 f. Dissertação (Mestrado em Biologia Animal) - Universidade Federal de Viçosa, Viçosa. 2009.

DYCE, K. M.; SACK, W. O.; WENSING, C. J. G. Tratado de Medicina Veterinária. 2. ed. Rio de Janeiro: Guanabara Koogan, 1996. $813 \mathrm{p}$. 
FERREIRA, A. O.; MORINI, A. C.; FAVARON, P. O.; PASSOS, C C.; CAMPOS, D. B.; MIGLINO, M. A.; GUERRA, R. R. Avaliação morfológica das membranas fetais e da placenta de Mazama gouazoubira (veado-catingueiro) de vida livre no terço inicial da gestação. Pesquisa Veterinária Brasileira, Rio de Janeiro, v. 31, n. 7, p. 631-635, 2011.

FIORETTO, E. T.; CASTRO, M. F. S.; GUIDI, W. L.; MAINARDI, R.; SOUZA R. R.; RIBEIRO, A. A. C. M. Gross anatomic organization of the capybara's (Hydrochaeris hydrochaeris) brachial plexus. Anatomy, Histology and Embryology, Berlin, v. 32, p. 169-174, 2003.

FREEMAN, P. M.; HARCOURT-BROWN, T. R.; JEFFERY, N. D.; GRANGER, N. J. Electrophysiologic evidence of polyneuropathy in a cat with signs of bilateral brachial plexus neuropathy. Journal of the American Veterinary Medical Association, Ithaca, v. 234, n. 2, p. 240-244, 2009.

FUTEMA, F.; FANTONI, D. T.; AULER-JR., J. O. C.; CORTOPASSI, S. R. G.; ACAUI, A.; STOPIGLIA, A. J. Nova técnica de bloqueio do plexo braquial em cães. Ciência Rural, Santa Maria, v. 29, n. 1, p. 63-69, 1999.

GAMBA, C. O.; CASTRO, T. F.; RICKES, E. M.; PEREIRA, M. A. M. Sistematização dos territórios nervosos do plexo braquial em chinchila (Chinchilla lanigera). Brazilian Journal of Veterinary Research and Animal Science, São Paulo, v. 44, n. 4, p. 283-289, 2007.

GETTY, R. Sisson - Grossman Anatomia dos animais domésticos. v. 1. 5. ed. Rio de Janeiro: Interamericana, 1981. 2000 p.

GHOSHAL, N. G. Nervos espinhais. In: GETTY, R. (Ed.). Anatomia dos animais domésticos. Rio de Janeiro: Guanabara Koogan, 1986. p. 1052-1077.

GHOSHAL, N. G. Nervos espinhais. In: GETTY, R. (Ed.). Anatomia dos animais domésticos. v. 1. 5. ed. Rio de Janeiro: Interamericana, 1996. p. 620-642.

GUIMARÃES, G. C.; MACHADO, M. R. F.; SANTOS, A. L. Q.; MIGLINO, M. A.; CATTELAN, J. W. Origem, ramificação e distribuição do nervo radial no braço do gato doméstico (Felis catus domesticus, Linnaeus 1758). Brazilian Journal of Veterinary Research and Animal Science, São Paulo, v. 44, n. 4, p. 261-267, 2007.

ICVGAN - INTERNATIONAL COMMITTEE ON VETERINARY GROSS ANATOMICAL NOMENCLATURE. Nomina Anatomica Veterinaria. 5. ed. Knoxville: World Association of Veterinary Anatomist, 2005. 190 p.

IWAMOTO, J.; YAMAGISHI, N.; SASAKI, K.; KIM, D.; DEVKOTA, B.; FURUHAMA, K. A novel technique of ultrasoundguided brachial plexus block in calves. Research in Veterinary Science, London, 2012. DOI: 10.1016/j.rvsc.2012.05.010. (In press).

KOIZUMI, M.; SAKAI, T. The nerve supply to coracobrachialis in apes. Journal of Anatomy, London, v. 186, n. 395-403, 1995.

KOIZUMI, M.; SAKAI, T. On the morphology of the brachial plexus of the platypus (Ornithorhynchus anatinus) and the echidna (Tachyglossus aculeatus). Journal of Anatomy, London, v. 190, p. 447-455, 1997.

LIMA, F. C.; SANTOS, A. L. Q., LIMA, B. C, VIEIRA, L. G.;
HIRANO, L. Q. L. Topographic anatomy of the spinal cord and vertebromedullary relationships in Mazama gouazoubira Fisher, 1814 (Artiodactyla; Cervidae). Acta Scientiarum. Biological Science, Maringá, v. 32, n. 2, p.189-194, 2010.

MELO, S. R.; GONÇALVES, A. F. N.; SASAHARA, T. H. C.; FIORETTO, E. T.; GERBASI, S. H.; MACHADO, M. R. F.; GUIMARÃES, G. C.; RIBEIRO, A. A. C. M. Sex-related macrostructural organization of the deer's brachial plexus. Anatomy, Histology and Embryology, Berlin, v. 36, p. 295-299, 2007.

MIGLINO, M. A.; SOUZA, W. M.; DIDIO, L. J. A. Sistema excretor do fígado de veados (Manzana americana, Blastocerus bezoarticus e Manzana simplicicornis). II. Sistematização do ramus principalis sinester. Brazilian Journal of Veterinary Research and Animal Science, São Paulo, v. 32, n. 4, p. 219-223, 1995.

MILLER, M.; CHRISTENSEN, G.; EVANS, H. Anatomy of the dog. Philadelphia: W. B. Saunders Company, 1964. 941 p.

MILLER, R. A. Comparative studies upon the morphology and distribution of the brachial plexus. The American Journal of Anatomy, New York, v. 54. p. 143-175, 1934.

MOURA, C. E. B.; ALBUQUERQUE, J. F. G.; MAGALHÃES, M. S.; SIlVA, N. B.; OLIVEIRA, M. F.; PAPA, P. C. Análise comparativa da origem do plexo braquial de catetos (Tayassu tajacu). Pesquisa Veterinária Brasileira, Rio de Janeiro, v. 27, n. 9, p. 357-362, 2007.

RIBEIRO, A. R.; PRADA, I. L. S.; BARROS, R. A. C.; SILVA, D. C. O. Origem do plexo braquial do macaco Cebus apella. Brazilian Journal of Veterinary Research and Animal Science, São Paulo, V. 42, n. 2, p. 143-149, 2005.

RIOJA, E.; SINCLAIR, M.; CHALMERS, H.; FOSTER, R. A.; MONTEITH, G. Comparison of three techniques for paravertebral brachial plexus blockade in dogs. Veterinary Anaesthesia and Analgesia, Oxford, v. 39, n. 2, p.190-200, 2012.

RODRIGUES, H. Técnicas anatômicas. 3 ed. Vitória: s.n., 2005. $269 \mathrm{p}$.

SANTANA, J. J.; ALBUQUERQUE, J. F. G.; MOURA, C. E. B.; COSTA, W. P.; OLIVEIRA, M. F.; BARRETO JÚNIOR, R. A.; MIGLIANO, M. A. Origem do plexo braquial de mocós (Kerodon rupestris Wied, 1820). Brazilian Journal of Veterinary Research and Animal Science, São Paulo, v. 40, n. 6, p. 391-396, 2003.

SANTOS, L. A.; SILVA, F. O. C.; EURIDES, D.; GUIMARÃES, E. C.; LIZARDO, F. B.; SOUSA, G. C.; FACURY NETO, M. A.; SROUR, H. A.; SANTOS, V. M. Origem e distribuição do nervo supra-escapular em fetos de bovinos azebuados. Biotemas, Florianópolis, v. 24, n. 1, p. 69-75, 2011.

SANTOS, L. A.; SILVA, F. O. C.; GUIMARÃES, E. C.; EURIDES, D.; LIZARDO, F. B.; SOUSA, G. C.; FACURY NETO, CABRAL, L. G.; SANTOS, V. M. Origem e distribuição do nervo toracodorsal em fetos de bovinos azebuados. Bioscience Journal, Uberlândia, v. 26, n. 6, p. 948-955, 2010.

SCAVONE, A. R. F.; MACHADO, M. R. F.; GUIMARÃES, G. C.; OLIVEIRA, F. S.; GERBASI, S. H. B. Análise da origem e distribuição dos nervos periféricos do plexo braquial da paca (Agouti paca Linnaeus, 1766). Ciência Animal Brasileira, Goiânia, v. 9, n. 
4, p. 1046-1055, 2008.

SOUZA, D. A. S.; CASTRO, T. F.; FRANCESCHI, R. C.; SILVA FILHO, R. P.; PEREIRA, M. A. M. Formação do plexo braquial e sistematização dos territórios nervosos em membros torácicos de lobos marinhos Arctocephalus australis. Brazilian Journal of Veterinary Research and Animal Science, São Paulo, v. 47, n. 2, p. 168-174, 2010.

STRODE, J. E. Brachial plexus block anesthesia: its advantages in the treatment of fractures of the arm: report of cases. California and Western Medicine, San Francisco, v. 31, n. 1, p. 17-20, 1929.

UZUN, A.; CENGÜZ, N.; KAVAKLI, A.; KARAKAS, S. Rat plexus brachialis'inin morfolojik ve mikroskobik üncelenmesi. Turkish Journal of Veterinary and Animal Sciences, Tubitak, v. 25, p. 397-402, 2001. 